\title{
A Multi-Functional Compact Button Antenna for Wearable Applications
}

\author{
Jiahao Zhang ${ }^{1}$, Sen $\mathrm{Yan}^{2}$, Xiaomu Hu${ }^{1}$, Tomislav Marinovic ${ }^{1}$, Guy A. E. Vandenbosch ${ }^{1}$ \\ ${ }^{1}$ ESAT-TELEMIC, KU Leuven, Leuven, Belgium, jiahao.zhang@esat.kuleuven.be \\ ${ }^{2}$ School of Electronics and Information, Xi'an Jiaotong University, Xi'an, China, sen.yan@xjtu.edu.cn
}

\begin{abstract}
A multi-functional button antenna is designed, with dual-band and dual-polarization characteristics. This antenna is a dedicated design for wearable applications. A compact size button is achieved with a diameter of only 19.5 $\mathrm{mm}(0.29 \lambda)$, which optimizes the users' comfort. The proposed antenna works in the 4.5-4.6 unlicensed future 5G communication band, and the 5.1-5.5 GHz WLAN band. Two radiation patterns with orthogonal linear polarizations are obtained in each band. The mutual coupling between the two patterns is below $-20 \mathrm{~dB}$. The antenna is prototyped. Simulations and experiments confirm the validity of this novel concept.
\end{abstract}

Index Terms-dipole antennas, button antennas, dual-band antennas, dual-polarized antennas, wearable antennas.

\section{INTRODUCTION}

Wireless body area networks (WBAN) have received more and more attention in recent years [1]-[3]. Wearable antennas are a crucial part of WBAN. They realize the wireless data transmission from on-body sensors to a computational center. Wearable antennas can be achieved in different categories, such as flexible substrate antennas [4], conductive textile antennas [5], button antennas [6], and so on.

Antennas with multiple functions are always desired in modern communication systems, since they include multiple standards and protocols. Dual-band antennas and dualpolarized antennas are very promising in WBAN systems. By using dual-polarized antennas, the polarization loss for example by human body movements can be overcome in WBAN. Wearable button-based multiband antennas have been studied in [6], [7], without polarization diversity. A button antenna with two different modes in two bands is proposed in [8]. However, the button is quite large, affecting the comfort of the user. Pattern diversity is not realized. Efforts also have been done to study a circularly polarized button to overcome the polarization loss [9]. The proposed antenna has only one band, which limits its application, and pattern diversity is not achieved.

In this paper, a multi-functional button antenna with dual-band and dual-polarization characteristics is designed. The proposed antenna is based on a grounded dipole topology. The radiator is loaded on a compact rigid PCB (button). A very small radiator size is obtained. The antenna ground is formed by a flexible conducting textile (clothes). A semi-rigid coaxial cable is used to feed the button antenna.
The proposed antenna works in the $4.5 \mathrm{GHz}$ and $5.2 \mathrm{GHz}$ band. Two radiation patterns with orthogonal polarizations are obtained.

\section{ButTON ANTENNA DESIGN}

The grounded crossed dipole topology is selected in this design. The antenna ground is formed by textile materials, which are easily integrated with clothes. The ground promotes a broadside radiation pattern. The radiator is located on a rigid PCB (button), which is fed by semiflexible coaxial cables. A miniaturized dipole is the basis. Additional structures are loaded on an inner meander dipole and an outer loop dipole, see Fig. 1. A slot connected to a copper folded structure, is introduced at the end of the outer loop dipole, see Fig. 1 (a). A meander is loaded to increase the current flow length for the inner dipole, see Fig. 1 (b). The inner and outer dipole are highly coupled. The induced currents increase the electrical length of both dipoles, see Fig. 1 (c). Dual-band operation is achieved by these two coupled radiator structures. A perpendicularly placed dualband dipole is added to obtain the orthogonal polarization.

A coupling feeding topology is used. A T-shaped coupling feed line on the bottom layer of the button PCB, is connected to the outer conductor of the coaxial cables. The inner conductor is soldered to the radiator, which is in the top layer of the button PCB.

The topology is given in Fig. 2. A button shaped rigid $1.524 \mathrm{~mm}$ thick RO4003C substrate is used to carry the radiator. A textile substrate (non-conductive $1.5 \mathrm{~mm}$ thick felt textile, with relative permittivity 1.4 and dielectric loss tangent 0.044 ) is used to mimic the cloth. The ground is a $0.17 \mathrm{~mm}$ thick ShieldIt textile with a conductivity of 118000

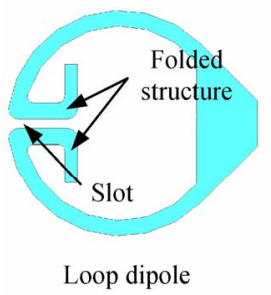

(a)

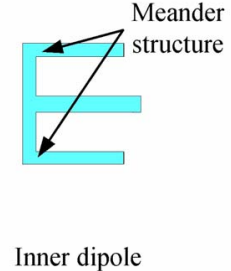

(b)

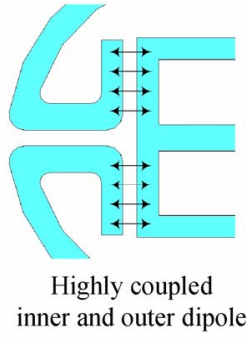

(c)
Fig. 1 (a) Loaded outer loop dipole. (b) Loaded inner dipole. (c) Highly coupled inner and outer dipole. 

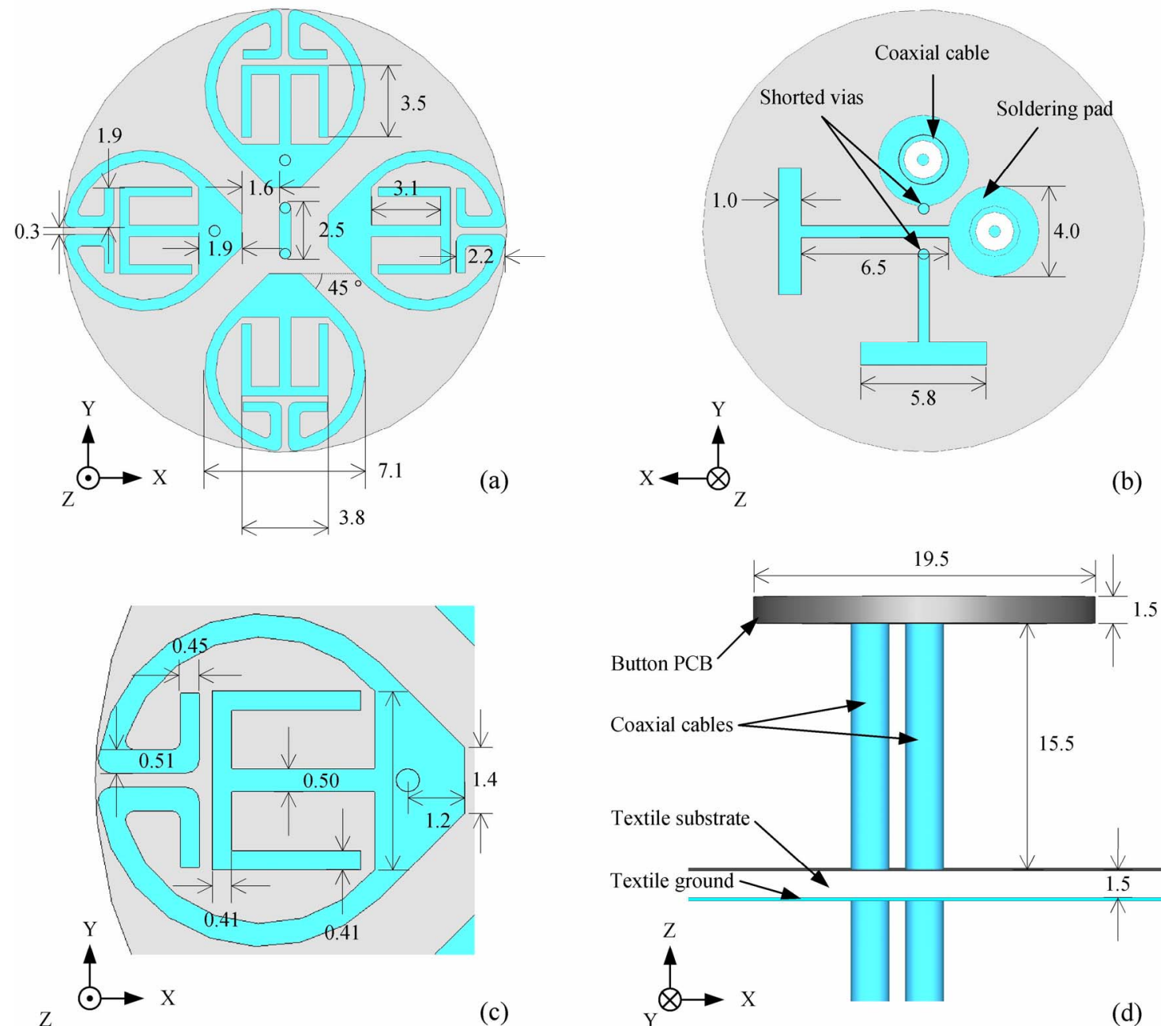

Fig. 2 Topology of the button antenna: (a) top view of the rigid substrate, (b) bottom view of the rigid substrate, (c) zoom of the radiator structure, (d) side view of the button antenna.

$\mathrm{S} / \mathrm{m}$. Two semi-rigid coaxial cables are used to feed the antenna. The outer conductors of the cables are soldered to the textile antenna ground. Note that the coaxial cables also act as supporters, and no additional structure is used to support the button PCB. This makes the proposed button antenna more realistic.

\section{Simulation AND MEASUREMENT RESUlts}

\section{A. Antenna Performance in Free Space}

The proposed antenna was prototyped and measured, see Fig. 3. The measured and simulated S-parameters are given in Fig. 4, showing a good agreement. Two resonant frequencies around $4.5 \mathrm{GHz}$ and $5.2 \mathrm{GHz}$ are observed for both ports. The measured overlapping bands are 4.50-4.61 $\mathrm{GHz}$ and 5.04-5.50 GHz, respectively.

The radiation patterns of port 1 are shown in Fig. 5. The front-to-back ratio (FBR) is above $15 \mathrm{~dB}$. The crosspolarization level is below $-10 \mathrm{~dB}$. The measured realized gain of port 1 and port 2 at $4.55 \mathrm{GHz}$ are 7.4 and $7.5 \mathrm{dBi}$, respectively, and at $5.2 \mathrm{GHz}$ they are 7.6 and $7.4 \mathrm{dBi}$, respectively. The corresponding simulated values are 7.8, 7.7, 7.6 , and $7.5 \mathrm{dBi}$, respectively. The measured total efficiencies of port 1 and port 2 at $4.55 \mathrm{GHz}$ are 86 and $85 \%$, respectively, and at $5.2 \mathrm{GHz}$ they are 93 and $92 \%$, respectively. The corresponding simulated values are 88,88 , 94 , and $94 \%$, respectively.

\section{B. Antenna Performance on Body}

The S-parameter performance of the antenna when bent is currently being studied. More detailed results concerning this will be presented and discussed at the conference.

\section{CONCLUSION}

A wearable button antenna is designed with a miniaturized radiator size. The antenna is based on a crossed dipole topology. A dual-band dual-polarized topology with the smallest radiator size in literature is achieved. The proposed antenna is able to cover two bands: the 4.50-4.61 $\mathrm{GHz}$ band and the 5.04-5.50 GHz band, and thus has a potential for $5 \mathrm{G}$, IoT, and WLAN applications. Two radiation patterns with orthogonal polarizations are achieved 


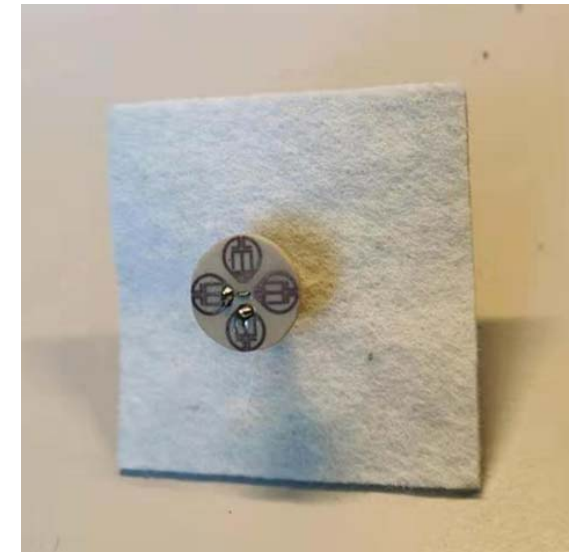

Fig. 3 Prototype.

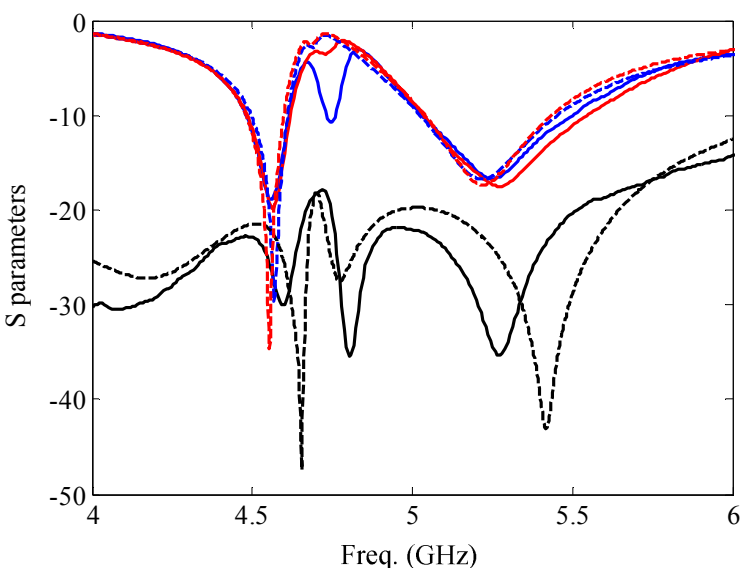

Fig. 4 S parameters, red lines $=|\mathrm{S} 11|$, blue lines $=|\mathrm{S} 22|$, black lines $=$ $|\mathrm{S} 21|$. Solid lines indicate measurements, dashed lines indicate simulations.

in both bands. The mutual coupling between the two patterns is below $-20 \mathrm{~dB}$. The button antenna is analyzed both in free space and on body. The simulated and measurement results indicate that the proposed antenna has a robust performance and is suitable for wearable applications.

\section{REFERENCES}

[1] P. Nepa, and H. Rogier, "Wearable antennas for off-body radio links at VHF and UHF bands: challenges, the state of the art, and future trends below $1 \mathrm{GHz}, "$ IEEE antennas and Propagation Magazine, vol. 57, no. 5, pp. 30-52, 2015.

[2] J. Zhang, S. Yan, and G. A. E. Vandenbosch, "A miniature feeding network for aperture-coupled wearable antennas," IEEE Transactions on Antennas and Propagation, vol. 65, no. 5, pp. 2650-2654, May 2017.

[3] J. Zhang, S. Yan, X. Hu, and G. A. E. Vandenbosch, "Mutual coupling suppression for on-body multi-antenna systems," IEEE Transactions on Electromagnetic Compatibility, early access.

[4] S. Ahmed, F. A. Tahir, A. Shamim, and H. M. Cheema, "A compact kapton-based inkjet-printed multiband antenna for flexible wireless devices," IEEE Antennas and Wireless Propagation Letters, vol. 14, pp. 1802-1805, 2015.

[5] C. Hertleer, H. Rogier, L. Vallozzi, and L. Van Langenhove, "A textile antenna for off-body communication integrated into protective
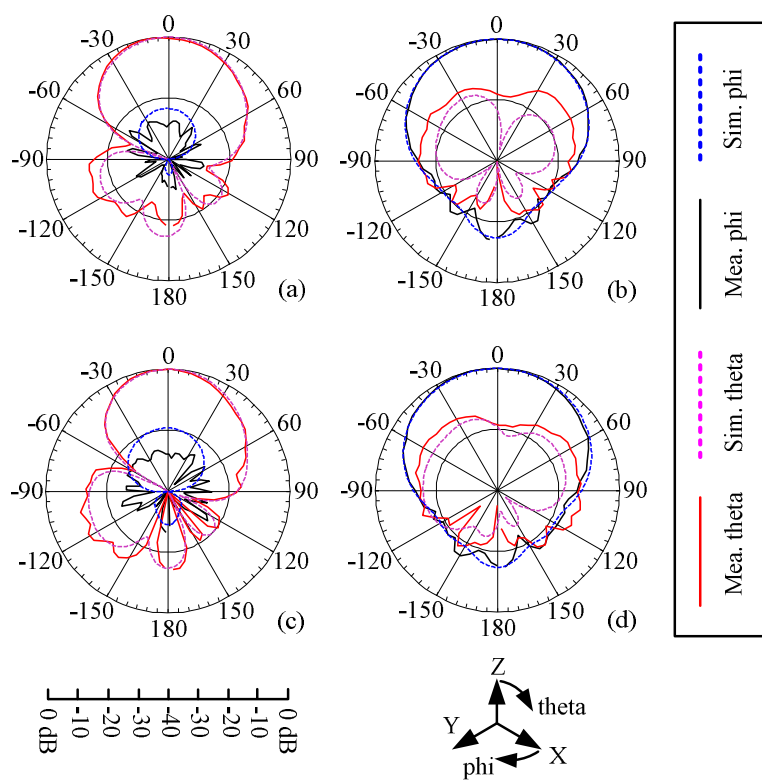

Fig. 5 Radiation patterns: Port 1 at $4.55 \mathrm{GHz}$, (a) XZ plane, (b) YZ plane. Port 1 at $5.2 \mathrm{GHz}$, (c) XZ plane, (d) YZ plane.

clothing for firefighters," IEEE Transactions on Antennas and Propagation, vol. 57, no. 4, pp. 919-925, 2009.

[6] B. Sanz-Izquierdo, F. Huang, J. C. Batchelor, "Covert dual-band wearable button antenna," Electronics Letters, vol. 42, no. 12, pp. 668-670, Jun. 2006.

[7] X. Hu, S. Yan, and G. A. E. Vandenbosch, "Wearable button antenna for dual-band WLAN applications with combined on and off-body radiation patterns," IEEE Transactions on Antennas and Propagation, vol. 65, no. 3, pp. 1384-1387, Mar. 2017.

[8] X. Y. Zhang, H. Wong, T. Mo, and Y. F. Cao, "Dual-band dual-mode button antenna for on-body and off-body communications," IEEE Transactions on Biomedical Circuits and Systems, vol. 11, no. 4, pp. 933-941, Aug. 2017.

[9] X. Hu, S. Yan, and G. A. E. Vandenbosch, "Compact circularly polarized wearable button antenna with broadside pattern for U-NII worldwide band applications," IEEE Transactions on Antennas and Propagation, vol. 67, no. 2, pp. 1341-1345, Feb. 2019. 